\title{
Schottky Solar Cells Based on Colloidal Nanocrystal Films
}

\author{
Joseph M. Luther, ${ }^{\dagger, \ddagger}$ Matt Law, ${ }^{\dagger}$ Matthew C. Beard, ${ }^{\dagger}$ Qing Song, ${ }^{\dagger}$ \\ Matthew 0. Reese, ${ }^{\dagger}$ Randy J. Ellingson, ${ }^{\dagger}$ and Arthur J. Nozik ${ }^{*, \dagger}$ \\ National Renewable Energy Laboratory, Golden, Colorado 80401, and Department of \\ Physics, Colorado School of Mines, Golden, Colorado 80401
}

Received August 13, 2008

\begin{abstract}
We describe here a simple, all-inorganic metal/NC/metal sandwich photovoltaic (PV) cell that produces an exceptionally large short-circuit photocurrent $\left(>21 \mathrm{~mA} \mathrm{~cm}^{-2}\right)$ by way of a Schottky junction at the negative electrode. The PV cell consists of a PbSe NC film, deposited via layer-by-layer (LbL) dip coating that yields an EQE of $55-65 \%$ in the visible and up to $25 \%$ in the infrared region of the solar spectrum, with a spectrally corrected AM1.5G power conversion efficiency of $2.1 \%$. This NC device produces one of the largest short-circuit currents of any nanostructured solar cell, without the need for sintering, superlattice order or separate phases for electron and hole transport.
\end{abstract}

Figure 1 shows the structure, current-voltage performance, EQE spectrum, and proposed band diagram of our device. Device fabrication consists of depositing a 60-300 nm-thick film of monodisperse, spheroidal $\mathrm{PbSe} \mathrm{NCs}$ onto patterned indium tin oxide (ITO) coated glass using a layer-by-layer dip coating method, followed by evaporation of a top metal contact. In this LbL method, ${ }^{1}$ a layer of NCs is deposited onto the ITO surface by dip coating from a hexane solution and then washed in $0.01 \mathrm{M}$ 1,2-ethanedithiol (EDT) in acetonitrile to remove the electrically insulating oleate ligands that originally solubilize the NCs (see Supporting Information). Large-area, crack-free and mildly conductive $(\sigma=5$ $\times 10^{-5} \mathrm{~S} \mathrm{~cm}^{-1}$ ) NC films result. The NCs pack randomly in the films, are partially coated in adsorbed ethanedithiolate, and show p-type conductivity under illumination. ${ }^{1} \mathrm{X}$-ray diffraction and optical absorption spectroscopy established that the NCs neither ripen nor sinter in response to EDT exposure. We have found that using methylamine instead of EDT yields similar device performance (Supporting Information, Figure 1). ${ }^{2}$ We have also fabricated working devices from $\mathrm{PbS}$ and $\mathrm{CdSe} \mathrm{NCs}$ (Supporting Information, Figures 2 and 3), which indicates that the approach adopted here is not restricted to EDT-treated PbSe NCs and that it should be possible to improve cell efficiency by engineering the surface of the NCs to attain longer carrier diffusion lengths and higher photovoltages through surface state passivation and prevention of Fermi level pinning.

* To whom correspondence should be addressed. E-mail: arthur_nozik@ nrel.gov.

$\dagger$ National Renewable Energy Laboratory.

$¥$ Colorado School of Mines.
When tested in nitrogen ambient under simulated 1-sun test conditions $\left(100 \pm 5 \mathrm{~mW} \mathrm{~cm}^{-2}\right.$ ELH white light illumination), EDT-treated PbSe devices exhibit large shortcircuit photocurrent densities $\left(J_{\mathrm{SC}}\right)$ and modest open-circuit voltages $\left(V_{\mathrm{OC}}\right)$ and fill factors $(\mathrm{FF})$, with one of the most efficient devices yielding $J_{\mathrm{SC}}=24.5 \mathrm{~mA} \mathrm{~cm}^{-2}, V_{\mathrm{OC}}=239$ $\mathrm{mV}, \mathrm{FF}=0.41$ and a mismatch-corrected ${ }^{3}$ AM1.5G efficiency of $2.1 \%$ (Figure 1a; see Supporting Information regarding spectral mismatch). The mismatch-corrected $J_{\mathrm{SC}}$ values of these devices are reproducibly larger than those of other nanostructured solar cells, including the best organic ${ }^{4}$ and dye-sensitized devices, ${ }^{5}$ which is remarkable considering the unsintered, glassy microstructure of our NC films and the fact that the NCs retain quantum confinement effects despite strong inter-NC carrier transport. The EQEs of the devices resemble the $\mathrm{NC}$ absorption spectra in the visible and near-infrared spectral regions and typically peak at $55-65 \%$ below $800 \mathrm{~nm}$ (Figure 1c). Taking into account the losses due to reflection and absorption by the electrodes, we estimate that the internal quantum efficiency (IQE) is about $80 \%$ in this part of the spectrum.

Multiple lines of evidence suggest that the photogenerated carriers in the device are separated by a Schottky barrier at the evaporated metal contact, as proposed in Figure $1 \mathrm{~d}$ and recently observed in films of PbS NCs. ${ }^{6}$ First, a linear fit to the data in Figure 2a shows that the opencircuit voltage $\left(V_{\mathrm{OC}}\right)$ of the cell increases with the $\mathrm{NC}$ bandgap, $E_{\mathrm{g}}$, as $V_{\mathrm{OC}} \approx 0.49\left(E_{\mathrm{g}} / q\right)-0.253 \mathrm{~V}$, where $q$ is the charge of an electron. The $V_{\mathrm{OC}}$ of a Schottky cell should scale linearly with the barrier height, $q \phi_{\mathrm{B}}$, and for an ideal contact between a metal and a $p$-type semiconductor, $q \phi_{\mathrm{B}}$ $=E_{\mathrm{g}}-q\left(\phi_{\mathrm{m}}-\chi\right)$, where $\phi_{\mathrm{m}}$ is the work function of the 

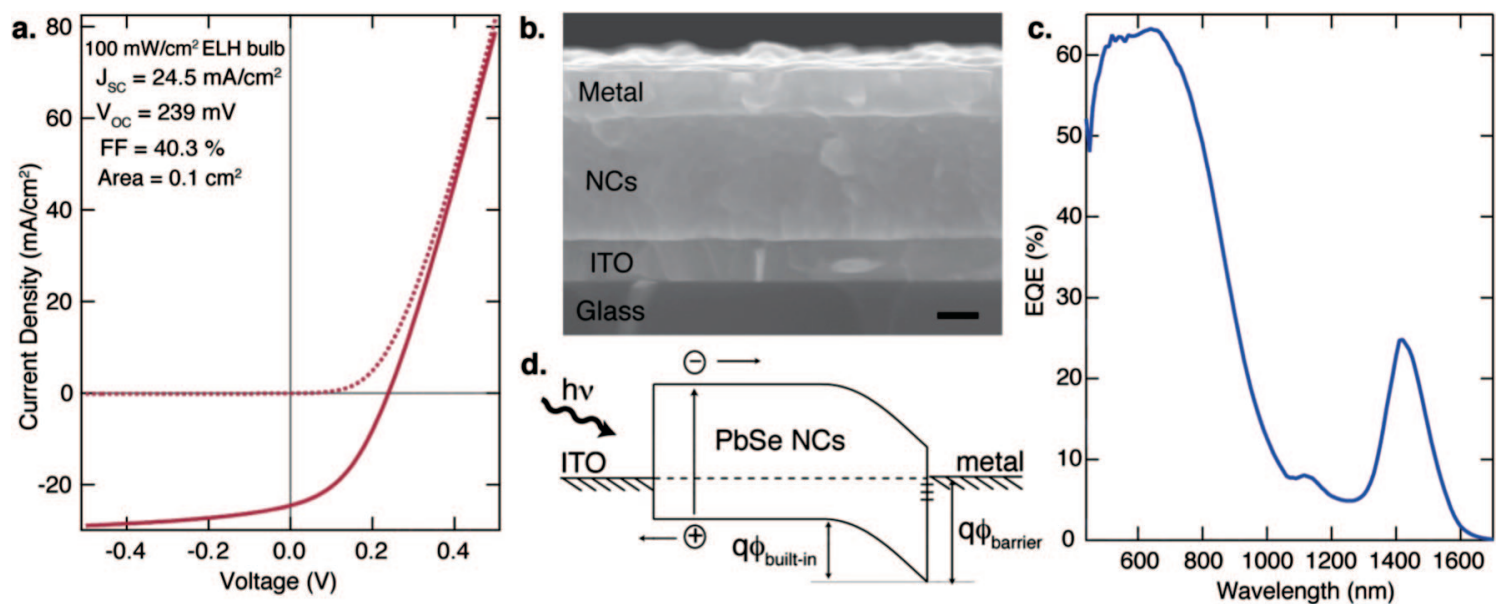

Figure 1. Structure, performance and schematic diagram of the device. (a) Current-voltage characteristics of a representative device in the dark and under $100 \pm 5 \mathrm{~mW} \mathrm{~cm} \mathrm{~cm}^{-2}$ simulated sunlight from an ELH tungsten halogen bulb $\left(E_{\mathrm{g}}\right.$ of $\left.\mathrm{NCs}=0.9 \mathrm{eV}\right)$. Correcting for the mismatch between the ELH and AM1.5G spectra yields $J_{\mathrm{SC}}=21.4 \mathrm{~mA} \mathrm{~cm}{ }^{-2}$ and an overall efficiency of $2.1 \%$ for this cell under $100 \mathrm{~mW}$ $\mathrm{cm}^{-2} \mathrm{AM} 1.5 \mathrm{G}$ illumination. The $J_{\mathrm{SC}}$ and $V_{\mathrm{OC}}$ show the usual linear and logarithmic dependence on light intensity (Supporting Information, Figure 4). (b) Scanning electron microscopy (SEM) cross-section of the ITO/NC film/metal device stack. The metal is $20 \mathrm{~nm}$ Ca/100 $\mathrm{nm}$ Al. The scale bar represents $100 \mathrm{~nm}$. (c) External quantum efficiency (EQE) of a different device with a $140 \mathrm{~nm}$-thick film $\left(E_{\mathrm{g}}=0.95 \mathrm{eV}\right)$. The first exciton transition of the NC film is seen at $1424 \mathrm{~nm}$. Integrating the product of the EQE and the AM1.5G spectrum from $350-1700$ $\mathrm{nm}$ yields $J_{\mathrm{SC}}=18.4 \mathrm{~mA} \mathrm{~cm}^{-2}$. (d) Proposed equilibrium band diagram. Light is incident through the ITO and band bending occurs at the interface between the NCs and evaporated negative electrode.
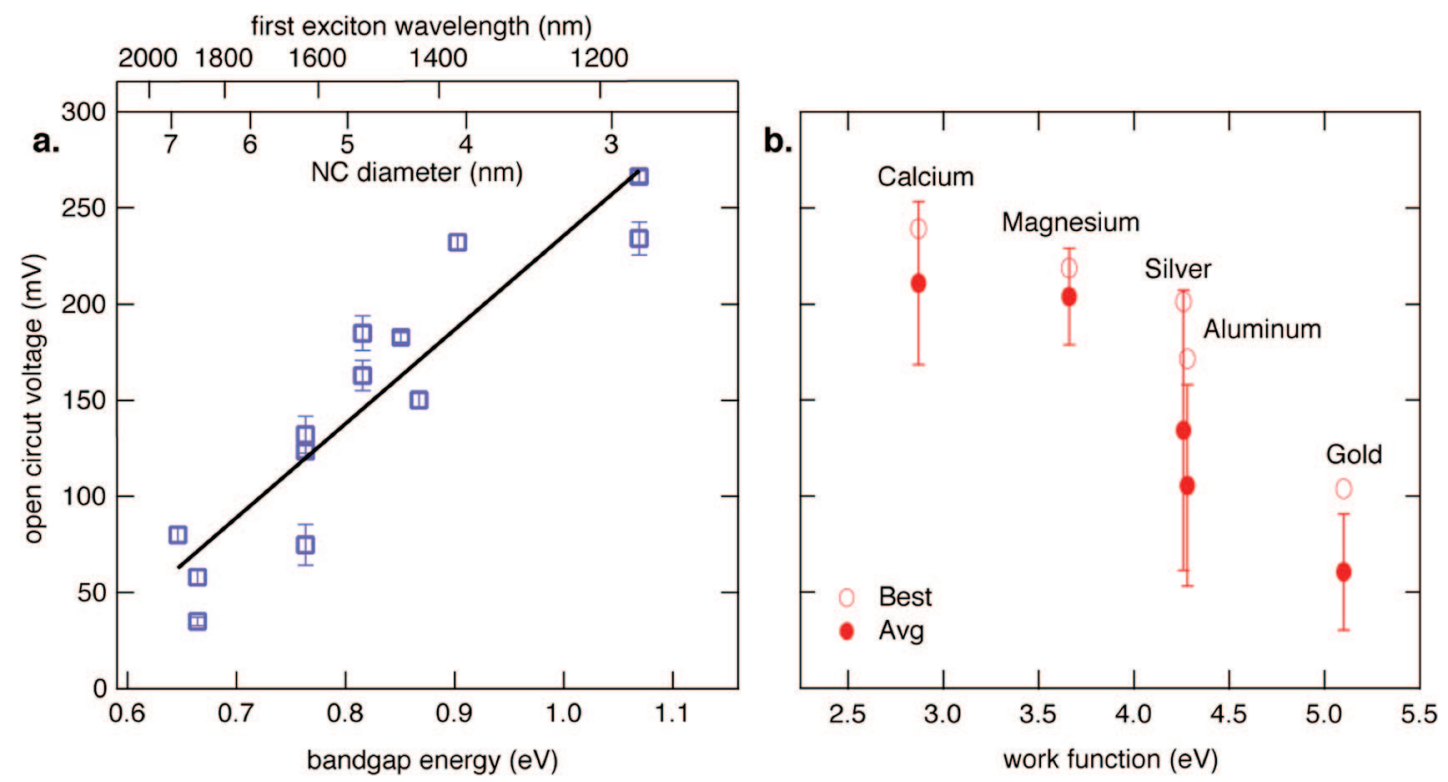

Figure 2. Trends in $V_{\mathrm{OC}}$ with NC size and metal work function. (a) Dependence on NC size. Each point is the average of six devices from a single substrate. The line is a least-squares fit to the data. (b) Dependence on metal work function $\left(E_{\mathrm{g}}=0.82 \mathrm{eV}\right)$. The best and average $V_{\mathrm{OC}}$ for each metal are shown, along with the standard deviation of six devices.

metal and $\chi$ the electron affinity of the semiconductor. ${ }^{7}$ In view of the similar electron and hole effective masses in $\mathrm{PbSe},{ }^{8}$ we expect equal displacement of the conduction and valence bands with increasing $E_{\mathrm{g}}$ and predict $\Delta V_{O C} \propto$ $0.5\left(\Delta E_{\mathrm{g}} / q\right)$ for a metal/PbSe junction, close to the experimental result. This dependence should hold even if the Fermi level is pinned at the interface by surface states, provided that the surface states do not shift appreciably in energy with changes in $E_{\mathrm{g}}$.

The second observation in support of the Schottky model is that the $V_{\mathrm{OC}}$ of the cell decreases with increasing work function of the top metal contact, as expected for a metal junction with a $p$-type semiconductor (Figure $2 \mathrm{~b}$ ). However, changing the contact metal from gold to calcium $\left(\left|\Delta \phi_{\mathrm{m}}\right|=\right.$ $2.3 \mathrm{eV}$ ) results in only a $0.15 \mathrm{~V}$ increase in $V_{\mathrm{OC}}$, which suggests that the surface Fermi level is pinned and the barrier height is relatively independent of the metal. Schottky barrier formation is often caused by defects formed at an interface by deposition of a metal, ${ }^{9}$ and this could easily be the case here. We note also that devices with gold top contacts do not switch polarity (that is, gold does not become the positive electrode) despite the fact that the work function of gold is substantially larger than that of ITO. Such a polarity reversal would be expected if our device were a metal-insulator-metal 

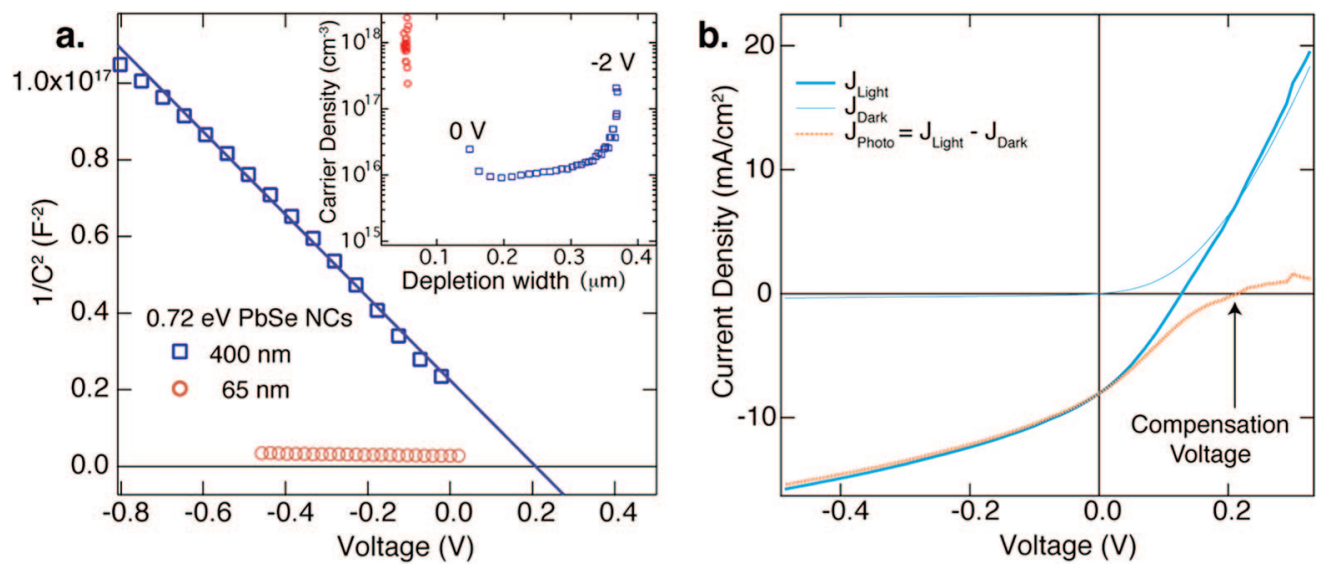

Figure 3. Analysis of the Schottky barrier. (a) Mott-Schottky plots at $1 \mathrm{kHz}$ for devices with a thin (65 nm, red) and thick (400 nm, blue) NC layer. The capacitance of the thin device is larger and changes little with reverse bias. A linear fit shows that the built-in potential of the thick device is $0.2 \mathrm{~V}$. Note that smaller NCs yield larger built-in potentials (not shown), as expected from Figure 2a. The inset shows the carrier concentration at the edge of the depletion layer for both devices. The thick device has an equilibrium depletion width of $\sim 150$ $\mathrm{nm}$, while the thin device is fully depleted. (b) $J-V$ characteristics of the thick device. The photocurrent $\left(J_{\text {Light }}-J_{\text {Dark }}\right)$ equals zero at a compensation voltage of $0.2 \mathrm{~V}$.

(MIM) type structure with a photovoltage driven by the difference in electrode work functions.

Direct evidence for the Schottky junction is obtained by capacitance-voltage $(C-V)$ measurements of complete cells. The simple structure of our device permits a determination of the built-in potential, depletion width, and carrier concentration of the NC film by Mott-Schottky analysis. The analysis assumes no free carriers in the depletion region and a carrier concentration outside the depletion region equal to the total acceptor density. The depletion width, $W$, of an abrupt Schottky junction is equal to

$$
W=\sqrt{\frac{2 \varepsilon \varepsilon_{0}\left(\phi_{\mathrm{bi}}-V\right)}{q N}}
$$

where $\varepsilon$ is the static dielectric constant of the NC film, $\varepsilon_{\phi}$ is the permittivity of free space, $\phi_{\mathrm{bi}}$ is the built-in potential, $V$ is the applied bias, and $N$ is the free carrier density at the edge of the depletion layer, given by

$$
N=\frac{1}{A^{2}} \frac{2}{q \varepsilon_{0} \frac{\mathrm{d}}{\mathrm{d} V}\left(\frac{1}{C^{2}}\right)}
$$

where $A$ is the device area and $C$ the capacitance. We used $\varepsilon=12$ for the NC films discussed here, as calculated with Bruggeman effective media theory (Supporting Information, Figure 5).

Mott-Schottky results for devices with a thin $(65 \pm 5$ $\mathrm{nm})$ and thick $(400 \pm 40 \mathrm{~nm}) \mathrm{NC}$ layer are presented in Figure 3a. The thick device acts as a well-behaved p-type diode with a built-in potential of $0.2 \mathrm{~V}$. The inset of Figure 3a shows $N$ as $W$ increases with applied reverse bias. The depletion width of the thick film is $150 \mathrm{~nm}$ at equilibrium and increases to $\sim 375 \mathrm{~nm}$ when the device nears full depletion at $\sim 1.7 \mathrm{~V}$ in reverse bias. Its equilibrium carrier density is determined to be $10^{16} \mathrm{~cm}^{-3}$. Since $W=150 \mathrm{~nm}$ at equilibrium for the thick device, the thin device (made from an identical NC film, only thinner) is fully depleted. The capacitance (and thus $W$ ) therefore does not change appreciably with reverse bias, and the carrier density cannot

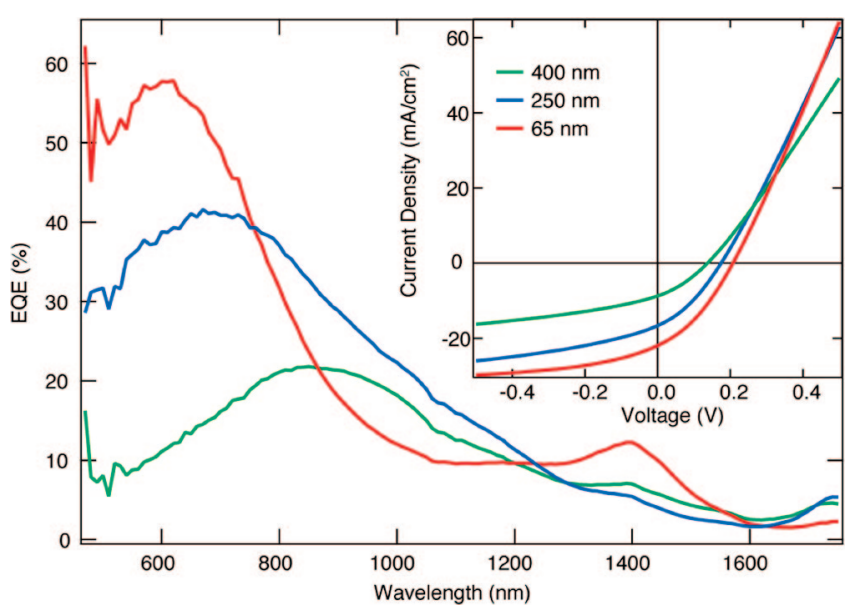

Figure 4. EQE dependence on NC film thickness. EQE spectra for devices with NC film thicknesses of $65 \pm 5,250 \pm 40$, and $400 \pm 40 \mathrm{~nm}\left(E_{\mathrm{g}}=0.72 \mathrm{eV}\right)$. The inset shows the corresponding $J-V$ traces at $100 \mathrm{~mW} \mathrm{~cm} \mathrm{~cm}^{-2}$ illumination.

be accurately determined from the slope of the Mott-Schottky plot. However, the data demonstrate that the measured capacitance corresponds to a width equivalent to the thickness of the thinner film and less than the zero-bias depletion width of the thick device.

The built-in potential found by $C-V$ measurements is in agreement with the estimate from $J-V$ plots. In a typical Schottky cell, the built-in potential is equivalent to the voltage at which the photocurrent $\left(J_{\text {Light }}-J_{\text {Dark }}\right)$ becomes zero. ${ }^{10}$ Figure $3 \mathrm{~b}$ shows that this occurs at $0.2 \mathrm{~V}$, in accord with the Mott-Schottky results of Figure 3a.

We deduced the location of the Schottky junction by comparing the EQE spectra from cells of different thickness. It is seen in Figure 4 that the EQE decreases markedly in the blue region of the spectrum as the thickness of the NC film is increased from 65 to $400 \mathrm{~nm}$. This falloff in the blue indicates that the charge-separating junction occurs at the back contact, at the interface between the NC film and the evaporated metal electrode. Because thicker devices have a 
wider field-free region near the ITO, blue photons, which are absorbed nearer the front of the cell $(1 / \alpha=75-100 \mathrm{~nm}$ at $\lambda=450 \mathrm{~nm}$ ), contribute progressively less to the photocurrent than do red photons, which penetrate closer to the depletion layer near the back contact. A back-contact Schottky geometry is consistent also with the polarity of the photocurrent and the $C-V$ analysis presented above.

Several additional observations can be made about the device based on the EQE and $J-V$ data of Figure 4. First, the diffusion length of excitons or carriers in the field-free region of the NC film must be fairly short, probably on the order of $100 \mathrm{~nm}$; if it were much longer, the blue EQE would decrease less sharply with increasing film thickness. Second, the $V_{\mathrm{OC}}$ is smaller for thicker devices because more carrier recombination occurs in the thicker films. The slope of the $J-V$ plots in forward bias indicates a higher series resistance for thicker devices, caused by the longer transport distance and rougher surface of the thicker NC films. The device shunt resistance $\left(d \mathrm{~V} / d \mathrm{~J}_{\mathrm{V}=0)}\right)$ increases modestly with film thickness.

Demonstrating an EQE above $100 \%$ at $h v=3 E_{\mathrm{g}}$ to $4 E_{\mathrm{g}}$ in a PbSe NC PV cell would provide unequivocal proof that multiple exciton generation ${ }^{11}$ (MEG), observed in many colloidal semiconductor $\mathrm{NCs}^{12-19}$ and in NC films, ${ }^{20}$ can be observed and exploited in devices. However, because our cells show a maximum EQE of only $\sim 65 \%$, we must rely on a determination of the IQE or a comparison of the shape of the EQE and optical absorption spectra to identify any anomalously large photocurrents that may be attributable to MEG. A proper analysis is complicated by strong optical interference effects in these thin cells as well as absorption by ITO at short wavelengths. A detailed optical and electrical model of the device parametrized by ellipsometry measurements has been developed to help answer this question. ${ }^{21}$ However, we have also recently found that the EDT treatment of our PbSe NC films, while enhancing their PV performance, suppresses MEG. ${ }^{22}$ Quenching of the MEG quantum yield by the EDT treatment potentially explains why the estimated IQE values reported here do not exceed 100\%.

There are a few drawbacks to the present PV device that deserve mention and future work. First, the Schottky junction is at the back rather than the front contact. This forces us to build relatively thin cells, which makes it difficult to achieve enough light absorption to yield higher EQEs and complicates our search for MEG photocurrent. Applying a small reverse bias during EQE measurements significantly enhances the photocurrent in the blue, but proper analysis of the photoconductive gain is required in order to determine whether the additional current originates from gain or MEG. Second, the device transforms from a diode to a $50 \Omega$ resistor when it is exposed to air for several minutes (Supporting Information, Figure 6). This degradation occurs in the dark, in the light, and with $\mathrm{Ca}$ or Au electrodes, and seems to result from surface oxidation of the nanocrystal film, which has been shown to dramatically increase film conductivity, ${ }^{1}$ combined with oxygen-induced loss of rectification at the NC-metal junction. We are now studying the mechanism of device degradation and developing the means to prevent oxidation of the NC film. Finally, as a Schottky cell our device can produce only a relatively small $V_{\mathrm{OC}}$, in theory little more than $0.5 E_{\mathrm{g}} / q^{23}$ A NC p-n or p-i-n structure would be superior in this respect.

We have introduced an all-inorganic PbSe $\mathrm{NC}$ solar cell that produces a large short-circuit photocurrent by fieldassisted separation of excitons and free carriers within a depletion region created near the interface of a $\mathrm{NC}$ film and a metal contact. This simple ITO/NC/metal device features a higher NC loading and fewer heterojunctions than either NCsensitized Gratzel or NC/polymer blend designs, and outperforms existing lead salt NC solar cells. ${ }^{24-34}$ Our work demonstrates that large EQEs are obtainable from NC cells without the need for sintering, superlattice order or separate phases for electron and hole transport. An improved understanding of electronic coupling, surface passivation, doping, and junction formation in NC films will lead to much more efficient and stable NC solar cells in the future.

Acknowledgment. The authors thank S. Kurtz, S. Shaheen, M. Hanna, J. van de Lagemaat, K. Emery, T. Moriarty, and D. Crandall for helpful discussions and D. Ginley for use of the fabrication glovebox. A.J.N., J.M.L., M.L., Q.S., M.C.B., R.J.E. were supported by U.S. DOE Office of Science, Division of Chemical Sciences and Nanoscience; M.O.R. was supported by the U.S. DOE EERE Photovoltaics Program; R.J.E. and J.M.L. were also partially supported by this program. The DOE work was funded by contract DEAC36-99-GO10337.

Supporting Information Available: Detailed information on materials and methods used to fabricate and characterize devices, Schottky junction devices using $\mathrm{PbS}$ and $\mathrm{CdSe} \mathrm{NCs}$, light intensity dependent $J V$ curves, and a discussion regarding the static dielectric constant of NC films. This material is available free of charge via the Internet at http:// pubs.acs.org.

\section{References}

(1) Luther, J. M.; Law, M.; Song, Q.; Perkins, C. L.; Beard, M. C.; Nozik, A. J. ACS Nano 2008, 2 (2), 271-280.

(2) Law, M.; Luther, J. M.; Song, Q.; Hughes, B. K.; Perkins, C. L.; Nozik, A. J. J. Am. Chem. Soc. 2008, 130 (18), 5974-5985.

(3) Shrotriya, V.; Li, G.; Yao, Y.; Moriarty, T.; Emery, K.; Yang, Y. Adv. Funct. Mater. 2006, 16 (15), 2016-2023.

(4) Mayer, A. C.; Scully, S. R.; Hardin, B. E.; Rower, M. W.; McGehee, M. D. Mater. Today 2007, 10 (11), 28-33.

(5) Gratzel, M. J. Photochem. Photobiol., A 2004, 164 (1-3), 3-14.

(6) Clifford, J. P.; Johnston, K. W.; Levina, L.; Sargent, E. H. Appl. Phys. Lett. 2007, 91 (25), 253117.

(7) Sze, S. Physics of Semiconductor Devices, 2nd ed.; John Wiley \& Sons: New York, 1981.

(8) Nimtz, G.; Schlicht, B. Narrow-Gap Semiconductors; Springer: Berlin, 1983.

(9) Schoolar, R. B.; Jensen, J. D.; Black, G. M. Appl. Phys. Lett. 1977, $31(9), 620-622$.

(10) Malliaras, G. G.; Salem, J. R.; Brock, P. J.; Scott, J. C. J. Appl. Phys. 1998, 84 (3), 1583-1587.

(11) Nozik, A. J. Physica E 2002, 14, 115-120.

(12) Schaller, R. D.; Klimov, V. I. Phys. Rev. Lett. 2004, 92 (18), 186601.

(13) Ellingson, R. J.; Beard, M. C.; Johnson, J. C.; Yu, P. R.; Micic, O. I.; Nozik, A. J.; Shabaev, A.; Efros, A. L. Nano Lett. 2005, 5 (5), 865871.

(14) Murphy, J. E.; Beard, M. C.; Norman, A. G.; Ahrenkiel, S. P.; Johnson, J. C.; Yu, P. R.; Micic, O. I.; Ellingson, R. J.; Nozik, A. J. J. Am. Chem. Soc. 2006, 128 (10), 3241-3247.

(15) Schaller, R. D.; Sykora, M.; Jeong, S.; Klimov, V. I. J. Phys. Chem. 
B 2006, 110 (50), 25332-25338.

(16) Beard, M. C.; Knutsen, K. P.; Yu, P. R.; Luther, J. M.; Song, Q.; Metzger, W. K.; Ellingson, R. J.; Nozik, A. J. Nano Lett. 2007, 7 (8), 2506-2512.

(17) Pijpers, J. J. H.; Hendry, E.; Milder, M. T. W.; Fanciulli, R.; Savolainen, J.; Herek, J. L.; Vanmaekelbergh, D.; Ruhman, S.; Mocatta, D.; Oron, D.; Aharoni, A.; Banin, U.; Bonn, M. J. Phys. Chem. C 2007, 111 (11), 4146-4152.

(18) Schaller, R. D.; Pietryga, J. M.; Klimov, V. I. Nano Lett. 2007, 7 (11), 3469-3476.

(19) Trinh, M. T.; Houtepen, A. J.; Schins, J. M.; Hanrath, T.; Piris, J.; Knulst, W.; Goossens, A.; Siebbeles, L. D. A. Nano Lett. 2008, 8 (6), 1713-1718.

(20) Luther, J. M.; Beard, M. C.; Song, Q.; Law, M.; Ellingson, R. J.; Nozik, A. J. Nano Lett. 2007, 7 (6), 1779-1784.

(21) Law, M.; Beard, M. C.; Choi, S.; Luther, J. M.; Hanna, M. C.; Nozik, A. J. Nano Lett. Submitted, 2008.

(22) Beard, M. C.; Midgett, A.; Law, M.; Nozik, A. J. Unpublished work, 2008.

(23) Nelson, J. The Physics of Solar Cells; Imperial College Press: London, 2003.

(24) Dissanayake, D.; Hatton, R. A.; Lutz, T.; Giusca, C. E.; Curry, R. J.; Silva, S. R. P. Appl. Phys. Lett. 2007, 91 (13), 133506.

(25) Hoyer, P.; Konenkamp, R. Appl. Phys. Lett. 1995, 66 (3), 349-351.
(26) Fritz, K. P.; Guenes, S.; Luther, J.; Kumar, S.; Saricifitci, N. S.; Scholes, G. D. J. Photochem. Photobiol. A 2008, 195 (1), 39-46.

(27) Jiang, X. M.; Schaller, R. D.; Lee, S. B.; Pietryga, J. M.; Klimov, V. I.; Zakhidov, A. A. J. Mater. Res. 2007, 22 (8), 2204-2210.

(28) Klem, E. J. D.; MacNeil, D. D.; Cyr, P. W.; Levina, L.; Sargent, E. H. Appl. Phys. Lett. 2007, 90 (18), 183113.

(29) McDonald, S. A.; Konstantatos, G.; Zhang, S. G.; Cyr, P. W.; Klem, E. J. D.; Levina, L.; Sargent, E. H. Nat. Mater. 2005, 4 (2), 138-142.

(30) Watt, A. A. R.; Blake, D.; Warner, J. H.; Thomsen, E. A.; Tavenner, E. L.; Rubinsztein-Dunlop, H.; Meredith, P. J. Phys. D: Appl. Phys. 2005, 38 (12), 2006-2012.

(31) Zhang, S.; Cyr, P. W.; McDonald, S. A.; Konstantatos, G.; Sargent, E. H. Appl. Phys. Lett. 2005, 87 (23), 233101.

(32) Cui, D. H.; Xu, J.; Zhu, T.; Paradee, G.; Ashok, S.; Gerhold, M. Appl. Phys. Lett. 2006, 88 (18), 183111.

(33) Koleilat, G. I.; Levina, L.; Shukla, H.; Myrskog, S. H.; Hinds, S.; Pattantyus-Abraham, A. G.; Sargent, E. H. ACS Nano 2008, 2 (5), 833-840.

(34) Johnston, K. W.; Pattantyus-Abraham, A. G.; Clifford, J. P.; Myrskog, S. H.; MacNeil, D. D.; Levina, L.; Sargent, E. H. Appl. Phys. Lett. 2008, 92 (15), 151115.

NL802476M 